\title{
Investigation of Sensor Placement for Accurate Fall Detection
}

\author{
Periklis Ntanasis ${ }^{1\left({ }^{(}\right)}$, Evangelia Pippa ${ }^{1}$, Ahmet Turan Özdemir ${ }^{2}$, Billur Barshan ${ }^{3}$, \\ and Vasileios Megalooikonomou ${ }^{1}$ \\ ${ }^{1}$ Department of Computer Engineering and Informatics, University of Patras, \\ Rion, Patras, Greece \\ \{ntanasis, pippa, vasilis\} @ceid.upatras.gr \\ 2 Department of Electrical and Electronics Engineering, Erciyes University, \\ Melikgazi, 38039 Kayseri, Turkey \\ aturanderciyes.edu.tr \\ ${ }^{3}$ Department of Electrical and Electronics Engineering, Bilkent University, \\ Bilkent, 06800 Ankara, Turkey \\ billur@ee.bilkent.edu.tr
}

\begin{abstract}
Fall detection is typically based on temporal and spectral analysis of multi-dimensional signals acquired from wearable sensors such as tri-axial accelerometers and gyroscopes which are attached at several parts of the human body. Our aim is to investigate the location where such wearable sensors should be placed in order to optimize the discrimination of falls from other Activities of Daily Living (ADLs). To this end, we perform feature extraction and classification based on data acquired from a single sensor unit placed on a specific body part each time. The investigated sensor locations include the head, chest, waist, wrist, thigh and ankle. Evaluation of several classification algorithms reveals the waist and the thigh as the optimal locations.
\end{abstract}

Keywords: Fall detection - Fall classification - Wearable sensors · Sensor placement $\cdot$ Machine learning $\cdot$ Classification $\cdot$ Accelerometers $\cdot$ Gyroscopes

\section{Introduction}

Falls are a common cause of injury among elderly people. According to the World Health Organization, 28-35\% of people aged 65 and over fall at least once a year with serious consequences such as heavy injuries and even death. Additionally, the moments after a fall are very critical. Many people experience what is called the "long lie," a long period of immobility after a fall that can have serious complications in a person's health. Unless precautions are taken, the number of injuries and the costs associated with fall-related trauma will double in the near future [1]. Fall detection is therefore considered an extremely important aspect of healthcare.

The most challenging aspect of fall detection is the distinction between falls and Activities of Daily Living (ADLs) such as sitting, standing and walking since falls typically occur while performing daily activities. In particular, ADLs with high acceleration 
are often confused with falls. Misinterpreting a fall as an ADL can have serious effects on the subject's health [2]. Therefore, a fall detection system should be able to accurately and immediately distinguish falls from ADLs when they occur. This requires falls to be automatically detected in real time. Another challenge is to make the system as simple as possible, with low false-alarm rates. Subjects using the system should feel comfortable and their quality of everyday life should not be affected. Accurate, reliable and real time fall detection systems are therefore essential.

Significant research has been conducted in this field and various fall detection systems have been proposed in the recent years. Noury et al. [3] and Yu et al. [4] have investigated the principles of fall detection and reviewed early works on the subject. Fall detection approaches can be divided into two main categories: vision-based and wearable device (motion sensor)-based systems.

Several context aware systems that use devices such as cameras or infrared sensors to detect falls within an environment have been developed. Rougier et al. [5] used human shape deformation to track the person's silhouette in recordings taken from four cameras. Falls and ADLs were classified with 98\% accuracy. In [6], a human 3D bounding box was created and the Kinect infrared sensor was used to accurately detect falls without any prior knowledge of the environment. Olivieri et al. [7] used motion templates taken from a camera to recognize certain ADLs and detect falls, achieving 99\% recognition rate. However, these approaches have certain limitations; the system can only monitor activities within the environment and thus, outdoor activities are excluded, restricting the mobility of the user. Also, other people moving within the same environment might also confuse the system and trigger false alarms in some cases.

The use of wearable motion sensors has been preferred by many researchers. With the advances in micro electro-mechanical systems (MEMS) technology, sensors such as accelerometers, gyroscopes and magnetometers have been integrated within small motion sensor units. The units that contain the above sensors can be used to collect movement data and detect falls. They are compact, light, inexpensive and have low power consumption. They can be placed in the subject's pockets or be easily attached at different body parts without making the subject uncomfortable; thus, they make the analysis of outdoor activities possible. Different body parts have been proposed for the sensor placement that improve the accuracy with minimum intrusion to the subject's everyday life. Yang and Hsu [8] have examined the fundamentals of such sensors as well as the optimal position on the human body for sensor placement.

In fall detection studies, typically simple thresholding is used. A fall is detected when the acceleration suddenly increases due to the change in orientation from upright to lying position [9]. In [10], the results of certain threshold-based methods that consider fall impact, velocity and posture have been assessed and tested on elderly subjects, achieving 94.6\% sensitivity. Thresholding methods sometimes tend to miss "soft falls," meaning falls that might not exceed the threshold. Also, certain ADLs with high acceleration may exceed the threshold and be misclassified as falls.

The main classification problem is to distinguish falls from ADLs. Machine learning techniques have been used to achieve more reliable results. Every recorded movement in the fall and activity database [11] has its own pattern. By extracting features from the raw data, these patterns can be classified by different classification methods. Before raw 
data are given to different classifiers, they must be pre-processed using a windowing technique. Such a technique divides the sensor signal into smaller time segments (i.e., windows) and a classification algorithm is applied separately to each window, producing a classification result. After pre-processing, features from the time or spatial domain are extracted to feed trained classifiers such as artificial neural (ANN) or Bayesian networks (BN), support vector machines (SVMs), decision trees, k-nearest neighbors (k-NN), etc. Kerdegari et al. [12] used statistical features such as maximum, minimum, mean, range, variance and standard deviation extracted from a waist-worn tri-axial accelerometer to investigate the performance of various classifiers on fall detection. The multilayer perceptron yielded the best sensitivity (90.15\%). Özdemir and Barshan [11] added autocorrelation coefficients and discrete Fourier transform (DFT) coefficients extracted from data acquired by sensors placed at different body parts. Six classifiers (k-NN, SVM, ANN, least-squares method, Bayesian decision making, dynamic time warping) were used to assign a fall or ADL class label to the feature vectors concatenated from all sensors. All methods achieved higher than $97.47 \%$ and $93.44 \%$ sensitivity and specificity, respectively. Yuwono et al. [13] obtained data from a single waist-worn tri-axial accelerometer and extracted features using the Particle Swarm Optimization (PSO) clustering method. Then, they proceeded to classify the data, achieving above $98.6 \%$ sensitivity in detecting falls.

Earlier studies report conflicting results on the best location to carry a single fall detection device on the human body. Some studies report that the waist is the best place since it is close to the body's center of gravity [10,14], while some claim that the chest or the head is better [9, 15-17]. Several studies consistently agree that the arms and the legs are not suitable parts of the body to carry a fall detection device since they are usually associated with higher accelerations [16, 18]. Resolving this issue through experiments that follow standardized procedures will be a valuable contribution. Özdemir and Barshan [11] acquired data from sensors placed on six body parts including the head, chest, waist, wrist, thigh and ankle. In order to proceed with classification, the features extracted from each location are concatenated to a single feature vector leading to a high-dimensional feature space. However, fall detection often needs to be performed in real time which requires lighter processing that can be achieved either through dimensionality reduction or selection of a single sensor unit located at the optimal position.

In this work, we attempt to determine the optimal location for the sensor placement on the human body. To achieve this, we evaluate the activity and fall dataset acquired by Özdemir and Barshan [11] with respect to several classification algorithms using only the data acquired from a single sensor location each time. The classification performance in terms of accuracy is used as the criterion to reveal the optimal sensor location. Since data from a single sensor unit are used, there is no need for dimensionality reduction, making the proposed methodology computationally efficient and thus, more capable of real-time fall detection.

The rest of the paper is organized as follows. In Sect. 2, we provide details on the dataset and the classification methodology. In Sect. 3, we present and discuss the achieved results. Finally, Sect. 4 concludes this work. 


\section{Materials and Methods}

\subsection{Dataset}

With Erciyes University Ethics Committee approval, seven males $(24 \pm 3$ years old, $67.5 \pm 13.5 \mathrm{~kg}, 172 \pm 12 \mathrm{~cm})$ and seven females $(21.5 \pm 2.5$ years old, $58.5 \pm 11.5 \mathrm{~kg}$, $169.5 \pm 12.5 \mathrm{~cm}$ ) healthy volunteers participated in the study with informed written consent. We tightly fitted six wireless sensor units with special strap sets to the subjects' heads, chests, waists, right wrists, right thighs, and right ankles. Each unit comprises three tri-axial devices (accelerometer, gyroscope, and magnetometer/compass) with respective ranges of $\pm 120 \mathrm{~m} / \mathrm{s}^{2}, \pm 1200 \%$, and $\pm 1.5 \mathrm{Gs}$, and an atmospheric pressure meter with 300-1100 hPa operating range, which we did not use. We recorded raw motion data along three perpendicular axes $(\mathrm{x}, \mathrm{y}, \mathrm{z})$ from each unit with a sampling frequency of $25 \mathrm{~Hz}$ [11]. A set of trials consists of 20 fall actions (front-lying, frontprotection-lying, front-knees, front-knees-lying, front-right, front-left, front-quickrecovery, front-slow-recovery, back-sitting, back-lying, back-right, back-left, rightsideway, right-recovery, left-sideway, left-recovery, syncope, syncope-wall, podium, rolling-out-bed) and 16 ADLs (lying-bed, rising-bed, sit-bed, sit-chair, sit-sofa, sit-air, walking-forward, jogging, walking-backward, bending, bending-pick-up, stumble, limp, squatting-down, trip-over, coughing-sneezing). We adopted these from [19] and each lasted about $15 \mathrm{~s}$ on the average. The 14 volunteers repeated each test for five times. Thus, we acquired a considerably diverse dataset comprising 1400 falls ( 20 tasks $\times 14$ volunteers $\times 5$ trials) and 1120 ADLs (16 tasks $\times 14$ volunteers $\times 5$ trials $)$, resulting in 2520 trials. Many of the non-fall actions included in the dataset are high-impact events that may be easily confused with falls.

\subsection{Feature Extraction}

Before we train the classifiers, we need to identify and isolate the actual experimental events since raw data acquired from the sensors include several time points that correspond to immobility before and after the detected fall event. In order to identify the fall event, we detect the peak of the total acceleration vector. Total acceleration is defined as:

$$
A_{T}=\sqrt{A_{x}^{2}+A_{y}^{2}+A_{Z}^{2}}
$$

where $A_{x}, A_{y}$ and $A_{z}$ are the accelerations along the $x, y$ and $z$ axis, respectively.

In contrast to [11] which considers the waist accelerometer as reference, we measure the total acceleration on each sensor unit separately. For each sensor type on the same unit, we keep two seconds of the sequence before and after the peak acceleration, that is, 50 values before and after the peak given the sampling frequency of $25 \mathrm{~Hz}$. Therefore, for each test, we obtain six arrays of size $9 \times 101$, one for each of the six sensor units.

We parameterize each one of the nine measured events using the features proposed in [11]: minimum, maximum and mean values, skewness, kurtosis, the first 11 values of the autocorrelation sequence and the first five frequencies with maximum magnitude of the DFT along with the five corresponding amplitudes, resulting in a feature 
vector of dimensionality 234 (26 features for each one of the nine measured signals) for each test.

\subsection{Classification}

We evaluate the ability of the above features to discriminate between falls and ADLs using several classification algorithms implemented by the WEKA machine learning toolkit [20] including J48 decision tree, k-nearest neighbors algorithm with value of $\mathrm{k}=7$ (IBk) [21], Random Forest (RF) [22, 23], Random Committee (RC) and SVM [24] with RBF Kernel (SMO). The classifiers in our study are selected in an attempt to evaluate representative algorithms for each one of the main categories of machine learning classifiers including decision trees (J48), support vector machines (SMO), ensemble classifiers (RF, RC) but also simple methods such as k-NN (IBk).

\section{Results}

We evaluated binary classification performance using accuracy, sensitivity and specificity. Evaluation was performed in a 10-fold cross validation setting.

Table 1 shows the achieved results in terms of accuracy, sensitivity and specificity for each sensor location for the J48, IBk, RC, RF and SMO algorithms, respectively. The position resulting in the best accuracy is highlighted in boldface fonts in the table. Figure 1 shows a comparative diagram across different body locations for each classifiers.

We achieve the overall highest accuracy (99.48\%) for the thigh sensor location using the SMO classifier. For this case, the obtained sensitivity, that is, the fraction of actual falls which are correctly identified as such is $99.21 \%$ and the specificity, that is, the proportion of ADLs that were correctly classified as such is $99.82 \%$. It seems that thigh-attached sensors can significantly reflect gait-related features during the performance of falls and ADLs, making their discrimination more accurate. The waist sensor location follows by achieving the highest accuracy values for the RF (99.28\%), RC (98.89\%) and k-NN, IBk (98.61\%) classifiers. These results agree with our intuition for the superiority of the waist location based on the fact that it is near the body's center of gravity. Finally, for the J48 classifier, the most accurate sensor location is the thigh, reaching $98.24 \%$ accuracy.

To summarize, the waist and thigh sensors achieve the highest accuracies for all classifiers, followed by the chest and ankle sensors. The wrist sensor is the one with the lowest accuracy for all classifiers, followed by the head. It is noteworthy, however, that all sensors achieve accuracies higher than $90 \%$ and there are cases where the differences among the sensors are not significant, especially when comparing the most accurate sensor locations such as the thigh and the waist. 
Table 1. Evaluation of the classifiers considered in this study

\begin{tabular}{|c|c|c|c|c|}
\hline Classifier & Sensors & Accuracy (\%) & Sensitivity (\%) & Specificity (\%) \\
\hline \multirow[t]{6}{*}{ (a) J48 } & Head & 96.48 & 91.06 & 95.76 \\
\hline & Chest & 97.53 & 97.70 & 97.31 \\
\hline & Waist & 97.96 & 97.99 & 97.94 \\
\hline & Wrist & 93.71 & 94.78 & 92.37 \\
\hline & Thigh & 98.24 & 98.71 & 97.67 \\
\hline & Ankle & 97.45 & 97.49 & 97.40 \\
\hline \multirow[t]{6}{*}{ (b) IBk } & \begin{tabular}{|l|} 
Head \\
\end{tabular} & 93.70 & 92.84 & 94.77 \\
\hline & Chest & 97.45 & 97.28 & 97.67 \\
\hline & Waist & 98.61 & 98.85 & 98.30 \\
\hline & Wrist & 89.74 & 84.13 & 96.77 \\
\hline & Thigh & 96.42 & 94.20 & 99.19 \\
\hline & Ankle & 95.58 & 93.34 & 98.39 \\
\hline \multirow[t]{6}{*}{ (c) $\mathrm{RC}$} & Head & 97.17 & 98.57 & 95.41 \\
\hline & Chest & 98.61 & 99.07 & 98.03 \\
\hline & Waist & 98.89 & 99.28 & 98.39 \\
\hline & Wrist & 94.63 & 96.35 & 92.47 \\
\hline & Thigh & 98.77 & 99.00 & 98.48 \\
\hline & Ankle & 98.77 & 98.85 & 98.66 \\
\hline \multirow[t]{6}{*}{ (d) $\mathrm{RF}$} & Head & 96.77 & 99.36 & 93.51 \\
\hline & Chest & 98.61 & 99.28 & 97.76 \\
\hline & Waist & \begin{tabular}{|l|}
99.28 \\
\end{tabular} & 99.64 & 98.84 \\
\hline & Wrist & 95.62 & 98.28 & 92.29 \\
\hline & Thigh & 99.20 & 99.43 & 98.93 \\
\hline & Ankle & 98.77 & 99.07 & 98.39 \\
\hline \multirow[t]{6}{*}{ (e) $\mathrm{SMO}$} & Head & 97.29 & 97.92 & 96.49 \\
\hline & Chest & 98.89 & 99.28 & 98.39 \\
\hline & Waist & 99.36 & 99.50 & 99.19 \\
\hline & Wrist & 96.78 & 97.71 & 95.61 \\
\hline & Thigh & 99.48 & 99.21 & 99.82 \\
\hline & Ankle & 98.57 & 98.85 & 98.21 \\
\hline
\end{tabular}

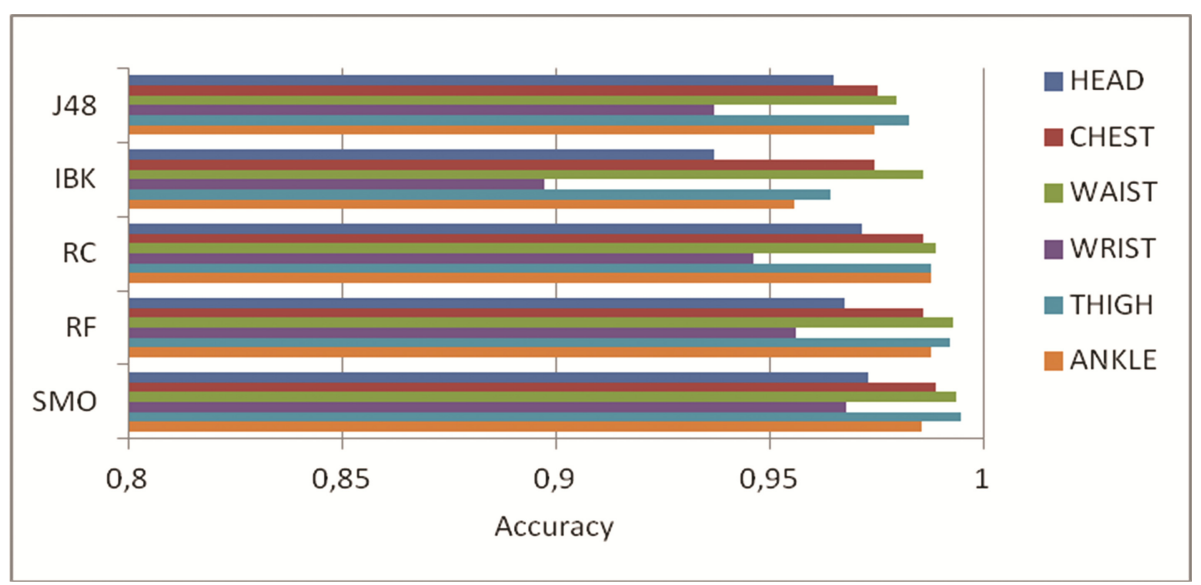

Fig. 1. Bar graph showing the accuracy of the classifiers considered for each sensor unit. 


\section{Conclusion}

In this paper, we investigated optimal sensor placement location for accurate fall detection based on feature extraction and classification. Evaluation of several classifiers reveals the superiority of thigh and waist locations. However, the differences on sensitivity and accuracy among the different sensor locations are relatively low and sometimes negligible, especially for the best performing waist and thigh sensors. Finally, since our method proposes the utilization of a single sensor unit, it keeps the feature vector dimensionality rather low, providing the means for real-time fall detection, even when using mobile devices with limited computational capabilities. In future work, a cross analysis can be conducted using actigraphy, to monitor daily activities and to detect and classify falls.

Acknowledgements. This work was supported by the FrailSafe project funded from the European Union's Horizon 2020 research and innovation programme under grant agreement No. 690140. The paper reflects only the view of the authors and the Commission is not responsible for any use that may be made of the information it contains.

\section{References}

1. World Health Organization: Global report on falls prevention in older age. http:// www.who.int/ageing/publications/Falls_prevention7March.pdf

2. Gurley, R.J., Lum, N., Sande, M., Lo, B., Katz, M.H.: Persons found in their homes helpless or dead. N. Engl. J. Med. 334, 1710-1716 (1996)

3. Noury, N., Fleury, A., Rumeau, P., Bourke, A.K., Laighin, G.O., Rialle, V., Lundy, J.E.: Fall detection-principles and methods. In: Proceedings of the 29th Annual International Conference of the IEEE Engineering in Medicine and Biology, Lyon, France, pp. 1663-1666 (2007)

4. Yu, X.: Approaches and principles of fall detection for elderly and patient. In: 10th International Conference on e-health Networking, Applications and Services, HealthCom, Singapore, pp. 42-47 (2008)

5. Rougier, C., Meunier, J., St-Arnaud, A., Rousseau, J.: Robust video surveillance for fall detection based on human shape deformation. IEEE Trans. Circuits Syst. Video Technol. 21, 611-622 (2011)

6. Mastorakis, G., Makris, D.: Fall detection system using Kinect's infrared sensor. J. Real-Time Image Proc. 9, 635-646 (2012)

7. Olivieri, D.N., Conde, I.G., Sobrino, X.A.V.: Eigenspace-based fall detection and activity recognition from motion templates and machine learning. Expert Syst. Appl. 39, 5935-5945 (2012)

8. Yang, C., Hsu, Y.: A review of accelerometry-based wearable motion detectors for physical activity monitoring. Sensors 10, 7772-7788 (2010)

9. Bourke, A.K., O’Brien, J.V., Lyons, G.M.: Evaluation of a threshold-based tri-axial accelerometer fall detection algorithm. Gait Posture 26, 194-199 (2007) 
10. Bourke, A.K., van de Ven, P., Gamble, M., O’Connor, R., Murphy, K., Bogan, E., McQuade, E., Finucane, P., Laighin, G., Nelson, J.: Assessment of waist-worn tri-axial accelerometer based fall-detection algorithms using continuous unsupervised activities. In: Annual International Conference of the IEEE Engineering in Medicine and Biology, Buenos Aires, Argentina, pp. 2782-2785 (2010)

11. Özdemir, A.T., Barshan, B.: Detecting falls with wearable sensors using machine learning techniques. Sensors 14, 10691-10708 (2014)

12. Kerdegari, H., Samsudin, K., Ramli, A.R., Mokaram, S.: Evaluation of fall detection classification approaches. In: 4th International Conference on Intelligent and Advanced Systems (ICIAS), Kuala Lumpur, Malaysia, pp. 131-136 (2012)

13. Yuwono, M., Moulton, B.D., Su, S.W., Celler, B.G., Nguyen, H.T.: Unsupervised machinelearning method for improving the performance of ambulatory fall detection systems. Biomed. Eng. Online 11, 1-11 (2012)

14. Özdemir, A.T.: An analysis on sensor locations of the human body for wearable fall detection devices: principles and practice. Sensors 16, 1161 (2016)

15. Kangas, M., Konttila, A., Lindgren, P., Winblad, I., Jamsa, T.: Comparison of low-complexity fall detection algorithms for body attached accelerometers. Gait Posture 28, 285-291 (2008)

16. Kangas M., Konttila A., Winblad I., Jamsa T.: Determination of simple thresholds for accelerometry-based parameters for fall detection. In: 29th Annual International Conference of the IEEE Engineering in Medicine and Biology Society, Lyon, France, pp. 1367-1370 (2007)

17. Bourke, A.K., Lyons, G.M.: A threshold-based fall-detection algorithm using a bi-axial gyroscope sensor. Med. Eng. Phys. 30, 84-90 (2008)

18. Bianchi, F., Redmond, S.J., Narayanan, M.R., Cerutti, S., Lovell, N.H.: Barometric pressure and triaxial accelerometry-based falls event detection. IEEE Trans. Neural Syst. Rehabil. Eng. 18, 619-627 (2010)

19. Abbate, S., Avvenuti, M., Corsini, P., Vecchio, A., Light, J.: Monitoring of human movements for fall detection and activities recognition in elderly care using wireless sensor network: a survey. In: Merret, G.V., Tan, Y.K. (eds.) Wireless Sensor Networks: Application-Centric Design, pp. 147-166. InTech, Rijeka (2010). Chapter 9

20. Hall, M., Frank, E., Holmes, G., Pfahringer, B., Reutemann, P., Witten, I.H.: The WEKA data mining software: An update. SIGKDD Explor. Newsl. 11, 10-18 (2009)

21. Aha, D.W., Kibbler, D., Albert, M.K.: Instance-based learning algorithms. Mach. Learn. 6, 37-66 (1991)

22. Liaw, A., Wiener, M.: Classification and regression by randomForest. R news 2, 18-22 (2002)

23. Breiman, L.: Random forests. Mach. Learn. 45, 5-32 (2001)

24. Keerthi, S.S., Shevade, S.K., Bhattacharyya, C., Murthy, K.R.K.: Improvements to Platt's SMO algorithm for SVM classifier design. Neural Comput. 13, 637-649 (2001) 